\title{
The tip of the iceberg: the frequency content of the $\delta$ Sct star HD 50844 from CoRoT space photometry
}

\author{
Poretti, E.*, Mantegazza, L.*, Rainer, M.*, Uytterhoeven, K., , Michel, E.**, \\ Baglin, A.**, Auvergne, M.**, Catala, C.**, Samadi, R.**, Rodríguez, E. ${ }^{*}$, \\ Garrido, R..$^{\ddagger}$ Amado, P. ${ }^{\ddagger}$, Martín-Ruiz, S. ${ }^{\ddagger}$, Moya, A. ${ }^{\ddagger}$, Suárez, J.C. ${ }^{\ddagger}$, Baudin, F. ${ }^{\S}$, \\ Zima, W. ${ }^{\mathbb{I}}$, Alvarez, M.॥, Mathias, P. ${ }^{\dagger \dagger}$, Paparó, M. ${ }^{\ddagger}$, Pápics, P. ${ }^{\ddagger}$ and Plachy, E. ${ }^{\ddagger}$ \\ ${ }^{*}$ INAF-OA Brera, Via E. Bianchi, 46, 23807 Merate, Italy \\ ${ }^{\dagger}$ Laboratoire AIM, CEA/DSM CNRS Université Paris Diderot, 91191, Gif-sur-Yvette, France \\ ${ }^{* *}$ LESIA, Observatoire de Paris, CNRS (UMR 8109), 5 pl. Janssen, 92195 Meudon, France \\ ¥Instituto de Astrofísica de Andalucía, Apartado 30040, 18080, Granada, Spain \\ ${ }^{\S}$ Institut d'Astrophysique Spatiale, CNRS, Université Paris XI UMR 8617, 91405, Orsay, France \\ II Instituut voor Sterrenkunde, K.U. Leuven, Celestijnenlaan 200 D, 3001 Leuven, Belgium \\ "Observatorio Astronómico Nacional, UNAM, Apto Postal 877, Ensenada, BC 22860, México \\ ${ }^{\dagger}$ UMR 6525 H. Fizeau, UNS, CNRS, OCA, Campus Valrose, F-06108 Nice Cedex 2, France \\ \# Konkoly Observatory, PO Box 67, 1525 Budapest, Hungary
}

\begin{abstract}
It has been suggested that the detection of a wealth of very low amplitude modes in $\delta$ Sct stars was only a matter of signal-to-noise ratio. Access to this treasure, impossible from the ground, is one of the scientific aims of the space mission CoRoT, developed and operated by CNES. This work presents the results obtained on HD 50844: the 140,016 datapoints allowed us to reach the level of $10^{-5} \mathrm{mag}$ in the amplitude spectra. The frequency analysis of the CoRoT timeseries revealed hundreds of terms in the frequency range $0-30 \mathrm{~d}^{-1}$. The initial guess that $\delta$ Sct stars have a very rich frequency content is confirmed. The spectroscopic mode identification gives theoretical support since very high-degree modes (up to $\ell=14$ ) are identified. We also prove that cancellation effects are not sufficient in removing the flux variations associated to these modes at the noise level of the CoRoT measurements. The ground-based observations indicate that HD 50844 is an evolved star that is slightly underabundant in heavy elements, located on the Terminal Age Main Sequence. The predominant term $\left(f_{1}=6.92\right.$ $\mathrm{d}^{-1}$ ) has been identified as the fundamental radial mode combining ground-based photometric and spectroscopic data.
\end{abstract}

Keywords: Stars: variables: $\delta$ Sct - Stars: oscillations - Stars: interiors

PACS: $97.10 . \mathrm{Sj}-$ 97.20.Ge - 97.30.Dg

\section{INTRODUCTION}

A huge effort has been made in the past decades to observe $\delta$ Sct stars from the ground. These opacity-driven pulsators show many excited modes with amplitudes that can be reached by photometry from the ground. After several results obtained by different teams performing single-site observations, the most promising targets have been monitored by means of multisite campaigns. A full description of the state-of-art in observing $\delta$ Sct stars from ground can be found in Breger \& Montgomery [2].

The CoRoT ${ }^{1}$ (COnvection, ROtation and planetary Transits) space mission gives us the possibility to investigate the pulsational content of $\delta$ Sct stars from a totally new perspective, i.e., high-precision measurements performed in a continuous way for tens of days (short runs) or more than one hundred days (Long Runs). One of the new $\delta$ Sct stars discovered in the preparatory work, HD 50844, was among the 10 asteroseismologic targets of the Initial Run (IR01), started on February $2^{\text {nd }}, 2007$ and finished on March $31^{\text {st }}, 2007(\Delta \mathrm{T}=57.61 \mathrm{~d})$. It is a slightly evolved star, close to the Terminal Age Main Sequence. The physical parameters derived from Strömgren photometry are $M_{V}=1.31, \mathrm{~T}_{\text {eff }}=7500 \mathrm{~K}, \log g=3.6$, and $[\mathrm{Fe} / \mathrm{H}]=-0.4$ dex [7]. In this contribution we summarize the results obtained from the analysis of the CoRoT data and the ground-based spectroscopy and photometry; Poretti et al. [8] give a more detailed description of all the procedures and discussions sketched here.

\footnotetext{
${ }^{1}$ The CoRoT space mission was developed and is operated by the French space agency CNES, with participation of ESA's RSSD and Science Programmes, Austria, Belgium, Brazil, Germany, and Spain.
} 


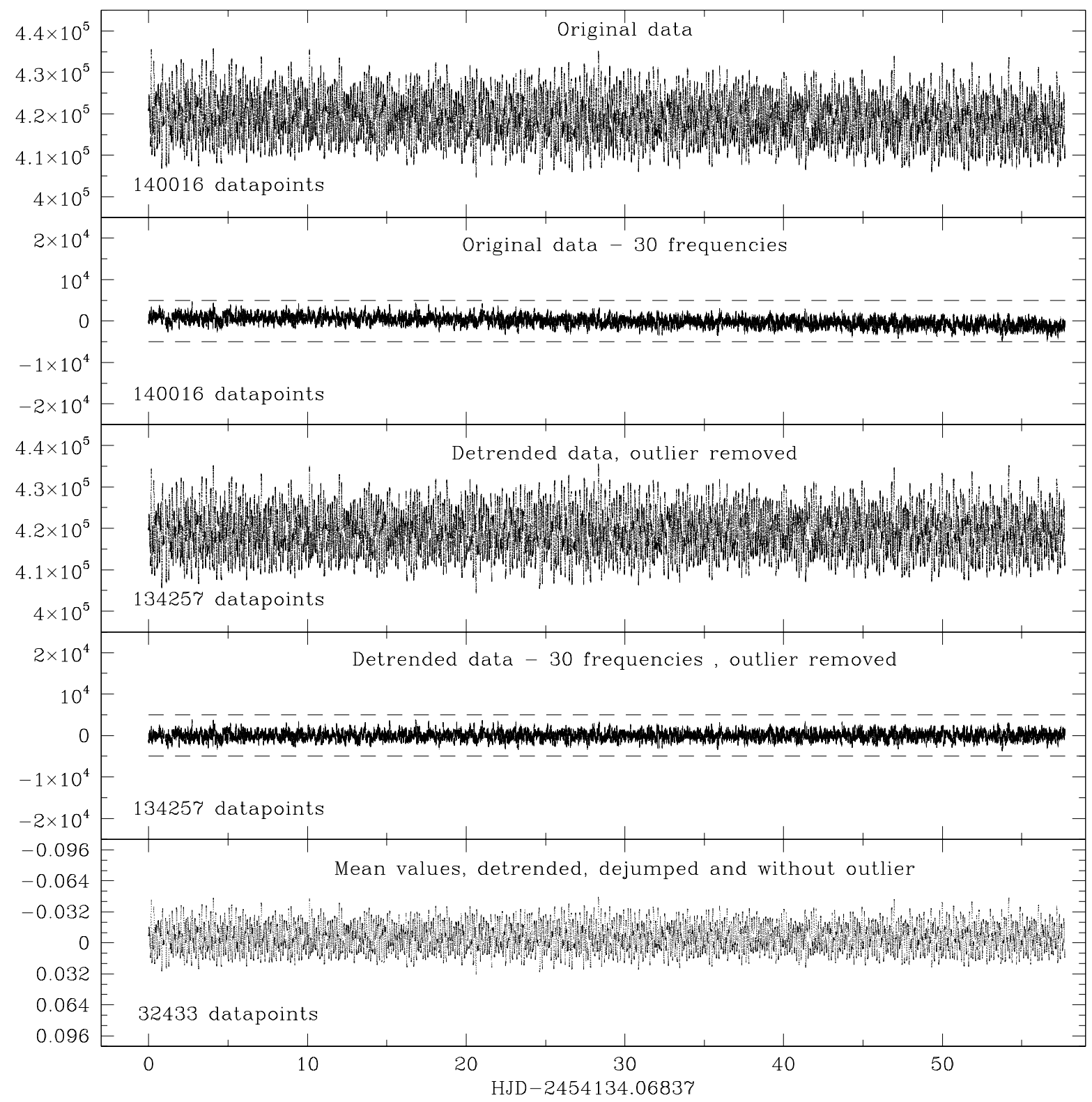

FIGURE 1. The different steps in preparing the final dataset of the CoRoT measurements of HD 50844. The measure units of the y-axes are observed flux for the first and third panels, residual flux for the second and fourth panels, magnitudes for the fifth panel. 


\section{THE REDUCTION OF THE COROT DATA}

For the analysis, we used the reduced N2 data rebinned at $32 \mathrm{sec}$ and we only considered the 140,016 datapoints for which no problem (i.e., flag=0) were reported (Fig.1, top panel). The rejection of the flagged points resulted in a slight enhancement of the amplitude of the orbital frequency, since the bad measurements occurred mostly when the satellite crossed the South Atlantic Anomaly (SAA). The light curve was detrended with a linear fit to remove the effect of ageing [1]. Firstly, the thirty frequencies with the highest amplitudes were identified and the data were prewhitened to clearly show outliers, jumps and long-term trend (Fig. 1 second panel). The final dataset is composed of 134,257 datapoints (Fig. 1 third panel) and the subsequent prewhitening leaves a residual light curve without appreciable trend (Fig. 1, fourth panel). To gain in CPU time and to reduce the noise level, in the last step we grouped the original data into new bins of four consecutive measurements, thus obtaining 32,433 datapoints (Fig. 1, bottom panel).

To have a reference frame for interpreting the results for HD 50844 (A2, $V=9.09$ ), we considered the data of HD 292790 (F8, $V=9.48$ ), which was observed by CoRoT in the same IR01. Our frequency analysis shows that the luminosity of HD 292790 is modulated by the rotation in a simple way. The amplitude spectrum shows the rotational frequency, its harmonics and the satellite frequencies (Fig. 2, top panel). For most part of the spectrum, i.e., from 10 to $100 \mathrm{~d}^{-1}$, the noise level is distributed in an uniform way and is very low, namely below $0.01 \mathrm{mmag}$. For $f<5 \mathrm{~d}^{-1}$, where the modulation terms and the long term drift are concentrated, the noise level slightly increases. On the other hand, the amplitude spectrum of HD 50844 appears to be very dense (Fig. 2 , second panel). At first glance, it is clear that the amplitude spectrum of HD 50844 is only for $f>50 \mathrm{~d}^{-1}$ as flat as that of HD 292790. We can calculate a noise amplitude of $7.5 \times 10^{-6} \mathrm{mag}$. It is straightforward to deduce that the signal is concentrated in the $f<30 \mathrm{~d}^{-1}$ domain, though there is no predominant peak region after identification of 250 frequencies (residual rms $1.2 \mathrm{mmag}$ ). At that point, the average peak height $(0.10 \mathrm{mmag})$ for $f<30 \mathrm{~d}^{-1}$ is still 10 times higher than for $f>50 \mathrm{~d}^{-1}$. A huge number of terms is necessary to reduce the amplitude spectrum of HD 50844 at the expected noise level. The peak height gets progressively lower after the identification of 500, 750, and 1000 terms (third, fourth, and fifth panels in Fig. 2, respectively). It is possible that some of the lower peaks originate from changes in amplitude or phase, or from other effects intrinsic to the star. In any case, the main conclusion of our analysis and related checks is that hundreds of terms - at least up to $1000-$
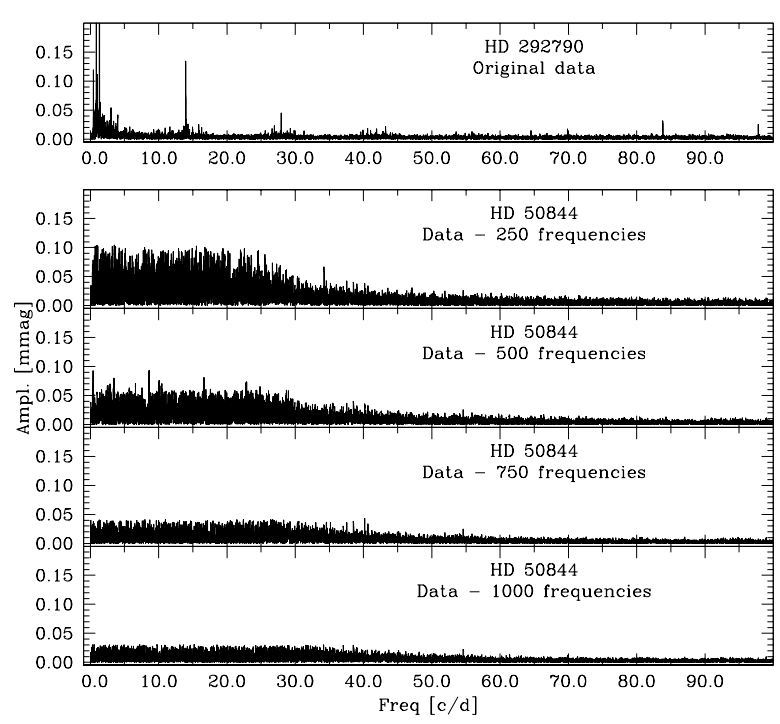

FIGURE 2. For comparison purposes, the top panel shows the amplitude spectrum of the rotational variable HD 292790. This star has been observed by CoRoT simultaneously to HD 50844. The amplitude spectra of HD 50844 shown in the other panels indicate the extreme richness of the pulsational spectrum of this $\delta$ Sct star: up to 1000 peaks are needed to make the residual spectrum of HD 50844 comparable to the original one of HD 292790.

are needed to explain the light variability of HD 50844. The detection of so many independent terms in the light variability of a $\delta$ Sct star is a totally new result.

Figure 3 shows the distribution of the first 100 frequencies identified in the CoRoT timeseries.

\section{MODE INDENTIFICATION FROM GROUND-BASED HIGH-RESOLUTION SPECTROSCOPY AND MULTICOLOUR PHOTOMETRY}

The spectroscopic observations were completed from January 2 to 28, 2007 with the FEROS instrument mounted at the 2.2-m ESO/MPI telescope, La Silla, Chile. The spectral resolution of FEROS is $R \sim 48,000$. We obtained 232 spectra in 14 nights. The exposure time was set to $900 \mathrm{sec}$ and the signal-to-noise ratios (SNRs) ranged from 150 to 210 . The spectra were reduced using an improved version of the standard FEROS pipeline, written in MIDAS and developed by Rainer [9]. The mean barycentric radial velocity of the star is $-10.8 \pm 0.2 \mathrm{~km} \mathrm{~s}^{-1}$. We also derived a $v \sin i$ value of $58 \pm 2 \mathrm{~km} \mathrm{~s}^{-1}$.

The spectroscopic frequencies were searched for by 


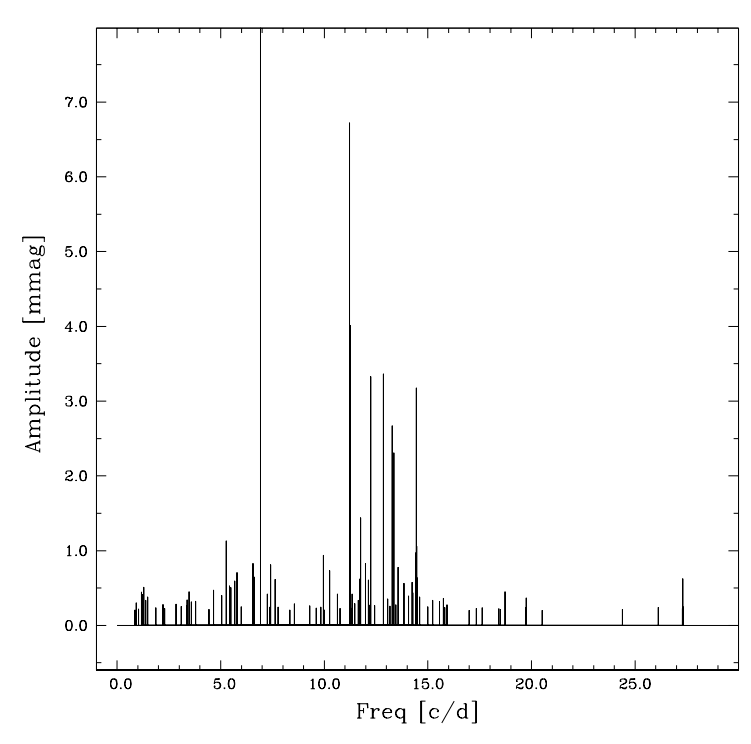

FIGURE 3. The amplitude spectrum of HD 50844, limited to the first 100 frequencies. The amplitude of the predominant term at $6.92 \mathrm{~d}^{-1}$ is out of scale (15.4 mmag).

analysing the LPVs by means of the pixel-by-pixel technique [5, 10]. We detected 27 terms with an SNR higher than 4.0 and 3 terms with $3.1<\mathrm{SNR}<3.5$. The mode identification was performed by fitting the amplitude and phase variations of each mode across the line profile [5] by using the software FAMIAS [11]. Uncertainties are estimated to be \pm 1 for the degree $\ell$ and \pm 2 for the order $m$.

The main goal of the spectroscopic observations is to answer the crucial question about the huge number of frequencies detected in the CoRoT photometric data: is the large number of photometric terms related to the visibility not only of low-degree modes but also of highdegree modes? To investigate this we focus on the mode identification results, as derived from the high-resolution data. The highest detected degree is $\ell=14$. This observational fact tells us that we have at least 235 possible modes of pulsation for a given radial order $n$. A few different radial orders are sufficient for explaining the 1000 frequencies detected in the CoRoT photometric timeseries of HD 50844. On the other hand, we know from CoRoT photometry that the frequency values higher than $30 \mathrm{~d}^{-1}$ are observed after the $300^{\text {th }}$ rank of detection, i.e., with very low amplitudes. The observed spectroscopic frequencies have $f<20 \mathrm{~d}^{-1}$. We still miss the spectroscopic counterpart of the $20<f<50 \mathrm{~d}^{-1}$ region. Such a region could be filled by modes with high radial orders $n$, which are shifted toward higher frequencies than those of the low orders.

In addition to the LPV analysis, the FEROS spectra were used to determine the abundances of the elements. HD 50844 is a slightly metal-deficient star. However, the abundances of elements as C, N, and S in the HD 50844 atmosphere are very similar to those of the Sun, while other elements are underabundant. Such an abundance pattern is typical of $\lambda$ Boo stars [6].

The importance of colour information to estimate the degree $\ell$ of the modes is well-known [4]; therefore, we observed HD 50844 in Stromgren uvby photometry to accompany the CoRoT white-light photometry. Several campaigns were carried out at S. Pedro Mártir and Sierra Nevada observatories, using twin Danish photometers. We calculated the amplitude ratios and the phase shifts by using the $y$ colour as the reference system. The immediate result is that the predominant term $f_{1}=6.92 \mathrm{~d}^{-1}$ is characterized by positive phase shifts (i.e., $\phi_{u, v, b}-\phi_{y}>$ 0.0 ). The mode identification of the $f_{1}$ term from the spectroscopic data was not trivial since we had to take the harmonic $2 f_{1}$ and the equivalent width variations into account. The FAMIAS method returns two possibile $(\ell, m)$ couples, i.e., $(0,0)$ and $(2,0)$. When considering both the spectroscopic and the photometric identifications, we can consider the $f_{1}$ term as the fundamental radial mode.

\section{CONCLUSIONS}

The exploitation of the CoRoT photometric timeseries of HD 50844 resulted in a very complex and intriguing task, also giving a completely new picture of the pulsational content of $\delta$ Sct stars. We demonstrated that the light curve of HD 50844 can be explained by the presence of hundreds of excited terms. Classical checks such as using different software packages, subdividing the timeseries into different subsets, carefully inspecting the residuals, and comparing with other similar targets observed by CoRoT, together confirm this issue. Highresolution spectra support the excitation of modes having a very high degree $\ell$ (up to $\ell=14$ ), thus providing an observational explanation for the richness of the frequency spectrum.

Such a large number of modes of different $\ell$ simultaneously excited is among plausible features suggested by theoretical works. An immediate conclusion is that the cancellation effects are not sufficient in removing the variations of the flux integrated over the whole stellar disk; Daszyńska-Daszkiewicz et al. [3] correctly predicted this extension to high degrees of the modes extracted from high-precision photometric timeseries. We should keep in mind that the CoRoT performances and the continuous monitoring allow detection of amplitudes of about $10^{-5}$ mag without significant aliasing effects, which is a totally new perspective.

The case of HD 50844 seems to match the theoretical picture featuring a very large number of excited modes 
with amplitudes limited by a saturation mechanism of the $\kappa$ mechanism. This confirms that the observation of $\delta$ Sct stars with CoRoT will allow us to address the longstanding problem of amplitude distribution and the potential existence of mode selection processes in the $\delta$ Sct instability strip. We note that HD 50844 probably does not constitute a "standard" scientific case of a $\delta$ Sct star, because it is located on the TAMS, probably belongs to the class of $\lambda$ Boo stars (i.e., showing atmospheric particularities), and it is seen almost equator-on. Nevertheless, seismic models that were able to reproduce the position of HD 50844 in the HR diagram and its measured rotational velocity were calculated.

HD 50844 has for the first time disclosed the intrinsic complexity of frequency spectra of intermediate-mass stars in the lower part of the instability strip. It is quite evident that, after these first CoRoT observations, we must look at $\delta$ Sct stars in a completely new way. We have to realize that ground-based observations were only able to observe the tip of the iceberg, while the larger part of the modes have remained undetected.

\section{ACKNOWLEDGMENTS}

The FEROS data are being obtained as part of the ESO Large Programme LP178.D-0361 (PI.: E. Poretti). Mode identification results were obtained with the software package FAMIAS developed in the framework of the FP6 Coordination Action Helio- and Asteroseismology (HELAS; http://www.helas-eu.org/). WZ was supported by the FP6 European Coordination Action HELAS and by the Research Council of the University of Leuven under grant GOA/2008/04. This work was supported by the Italian ESS project, contract ASI/INAF I/015/07/0, WP 03170, and by the Hungarian ESA PECS project No. 98022. KU acknowledges financial support from a $E u$ ropean Community Marie Curie Intra-European Fellowship, contract number MEIF-CT-2006-024476. JCS acknowledges support from the Consejo Superior de Investigaciones Cientificas by an I3P contract financed by the European Social Fund and from the Spanish Plan $\mathrm{Na}$ cional del Espacio under project ESP2007-65480-C0201. PJA acknowledges financial support from a Ramon y Cajal contract of the Spanish Ministry of Education and Science. AM ackowledges financial support from a Juan de la Cierva contract of the Spanish Ministry of Science and Technology. SMR acknowledges a Retorno de Doctores contract financed by the Junta de Andalucía and Instituto de Astrofísica de Andalucía for carrying out observing campaigns for CoRoT targets at Sierra Nevada Observatory. EN acknowledges financial support of the N N203 302635 grant from the MNiSW.

\section{REFERENCES}

1. Auvergne, M., Bodin, P., Boisnard, L., et al., 2009, A\&A, in press (arXiv:0901.2206)

2. Breger, M., Montgomery, M.H.., Editors, Delta Scuti and Related Stars, ASP Conf. Series, vol. 210

3. Daszyńska-Daszkiewicz, J., Dziembowski, W.A., \& Pamyatnykh, A.A., 2006, Mem. SAIt, vol. 77, 113

4. Garrido, R., 2000, in "Delta Scuti and Related Stars", M. Breger \& M.H. Montgomery Eds., ASP Conf. Series, 210, 67

5. Mantegazza, L., 2000, in "Delta Scuti and Related Stars", M. Breger \& M.H. Montgomery Eds., ASP Conf. Series, 210, 138

6. Paunzen, E., 2004, in "The A-Star Puzzle", J. Zverko, J. Ziznovsky, S.J. Adelman, \& W.W. Weiss Eds., Proc. IAU Symp. 224 (Cambridge University Press), p. 443

7. Poretti, E., Alonso, R., Amado, P.J., et al., 2005, AJ, 129, 2461

8. Poretti, E., Michel, E., Garrido, R., et al., 2009, A\&A in press (arXiv:0906.2628)

9. Rainer, M., 2003, Laurea Thesis (in Italian), Università degli Studi di Milano

10. Zima, W., 2006, A\&A, 455, 227

11. Zima, W., 2008, CoAst, 155, 17 